\title{
Why Another Focus on Melanoma?
}

To answer this question we have to look back on the past decades of development in diagnostics and therapy of melanoma, thereby acknowledging some milestones and realizing some deficiencies.

In humans, primary cutaneous malignant melanoma represents $1.5-2.5 \%$ of all malignant tumors. Therefore melanoma belongs to the group of rare neoplasias, although its incidence and mortality are permanently increasing. In Germany, incidence has quadrupled during the past decades to $10-12$ cases per 100,000 residents, i.e. 10,000 new melanoma patients per year. However, our experiences regarding diagnostic procedures and therapeutic options are characterized by contrasting extremes: we find ourselves between impressive success in basic research and disappointing results in the clinical reality. In the present issue, selected aspects of the management of patients suffering from melanoma are reviewed. They focus on some of the most important and promising new findings that deal with different facets of the care of patients with melanoma regarding surgical procedures, adjuvant therapy, palliation of advanced disease, and follow-up care.

A central part of melanoma treatment is the surgical resection of the primary tumor. The introductory article by Hauschild et al. [1] shows the influence of different resection safety margins and sentinel node biopsy on prognosis. During the past years, a consensus of recommendations for resection safety margins has been developing. The experience of recent decades has shown that the extend of surgical intervention can be reduced drastically and therefore patients are no longer impaired by $5 \mathrm{~cm}$ surgical margins and en-bloc lymph node dissections or even amputations. In contrast, surgical interventions nowadays result merely in $0.5-2 \mathrm{~cm}$ resection margins, elective lymph node dissection is no longer recommended and diagnostic sentinel node biopsy allows precise staging of the melanoma. Changing the surgical procedure was a milestone in treatment resulting in an essential reduction of therapyassociated immediate and long-term morbidity.
Despite intensive research and numerous clinical trials the impact of adjuvant treatment modalities on high-risk melanoma is still under debate. But even prospective randomized multicenter trials as yet failed to definitively identify a benefit concerning survival rate of melanoma patients. Mohr et al. [2] try to unravel and re-evaluate historical and current treatment concepts as well as new recommendations. At present, interferon - which is now licensed for adjuvant use - still represents the keystone of adjuvant therapy in high-risk melanoma, but the optimal dosage and duration of treatment are yet to be defined.

We have considerably increased our knowledge about the biological behavior of melanomas and have developed technologies and drugs to interfere with survival strategies of tumor cells. However, transfer from basic research to clinical practice is mostly hampered by multidrug resistance of the tumor cells, tumor escape mechanisms, and poor bioavailability of cytotoxic drugs at tumor sites. The broad spectrum of palliative treatment options reflects an impressive development in pharmacotherapy but it also shows that the breakthrough in the therapy of metastasizing melanoma is still missing. From painstaking study protocols the conclusion has to be drawn that most treatment concepts so far failed to be effective. In fact, no evidence-based advantage in survival could be shown for mono- or polychemotherapy or multimodal treatment, although immunochemotherapy has been shown to increase the relapse-free time interval and in some patients even results in long-time survival. However, thirty years after the development and licensing of dacarbazine, this drug still is considered the 'gold standard' with a success rate of about $20 \%$.

New concepts to improve this situation are reviewed by Ugurel and Schadendorf [3]. They discuss some innovative experimental treatment modalities for advanced disease that are currently investigated in clinical trials, such as chemosensitization by downregulation of bcl-2, vaccination strategies with

\begin{tabular}{ll}
\hline KARGER & ( ) 2003 S. Karger GmbH, Freiburg \\
$\begin{array}{ll}\text { Fax +497614520714 } & \text { Accessible online at: } \\
\text { E-mail Information@Karger.de } \\
\text { www.karger.com }\end{array}$ & www.karger.com/onk \\
&
\end{tabular}

Prof. Dr. Wolfgang Tilgen

Universitätskliniken des Saarlandes

Hautklinik und Poliklinik

D-66421 Homburg/Saar

Tel. +49 6841 1623-800, Fax -845

E-mail hawtil@uniklinik-saarland.de 
autologous peptide-pulsed dendritic cells, and immunomodulation by histamine. The authors point out the particular importance of chemoresistance of melanoma cells for the disappointing treatment results and focus on strategies to overcome these mechanisms. In this context, pretherapeutic chemosensitivity testing may be a tool to tailor and individualize cancer treatment.

Besides developing new concepts for the treatment of malignant melanoma, we have learned to manage side effects of systemic therapy. For example, the suppression of chemotherapy-induced vomiting and nausea by 5-HT3-receptor antagonists can be considered an important step to increase the quality of life of patients suffering from metastasizing malignant melanoma. Furthermore, we were successful in primary and secondary prevention, that means fair-skinned people have changed - at least in part - their ideal of beauty and prefer to remain pale instead of getting tanned. Patients were taught self-examination. Self-responsibility together with prevention efforts including identification and screening of risk groups have led to early detection of thin melanoma. An important 'take home message' is that early recognition of melanoma presents the best chance for cure. The frequently unpredictable course of advanced disease is illustrated by the case report by Fink et al. [4] on a patient who suffered from a melanoma which metastasized to common and uncommon sites of mucous membranes.
Garbe and Schadendorf [5] convincingly demonstrate an urgent need for re-orientation of the extensive diagnostic procedures performed routinely in the follow-up care of melanoma patients, which are only of limited benefit. Therefore diagnostic procedures should be prognosis-orientated: less intense in patients at low risk and more intense in patients at high risk of recurrent disease. Consensus guidelines for the management and follow-up examinations were developed and discussed according to published guidelines from other countries. These new recommendations are believed to result in physical and psychological benefit for patients and to reduce the continuously rising costs for the health system.

In conclusion: there has been considerable movement in the treatment and follow-up of this complicated disease - that's why we needed another focus on melanoma.

W. Tilgen, Homburg

\section{References}

1 Hauschild A, Rosien F, Lischner S: Surgical standards in the primary care of melanoma patients. Onkologie 2003;26:218-222.

2 Mohr P, Weichenthal M, Hauschild A: Adjuvant therapy in melanoma. Onkologie 2003;26:227-233.

3 Ugurel S, Schadendorf D: Systemic treatment in advanced melanoma: Innovative perspectives. Onkologie 2003;26:234-238.

4 Fink W, Zimpfer A, Ugurel S: Mucosal metastases in malignant melanoma. Onkologie 2003;26:249-251.

5 Garbe C, Schadendorf D: Surveillance and follow-up examinations in cutaneous melanoma. Onkologie 2003;26:241-246. 